\section{Case Reports in Ophthalmology}

\title{
Acute Anterior Uveitis in a Patient Taking Fingolimod (FTY720) for Multiple Sclerosis
}

\author{
Heather Gwen Mack ${ }^{\text {a-d }}$ Melissa Chih-Hui Tien ${ }^{\mathrm{e}}$ Owen Bruce White ${ }^{\mathrm{a}, \mathrm{b}}$ \\ ${ }^{a}$ Cabrini Medical Centre, Malvern, VIC, Australia; ${ }^{b}$ Melbourne Health,

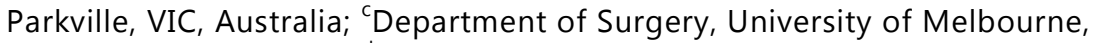 \\ Parkville, VIC, Australia; ${ }^{d}$ Walter and Eliza Hall Institute of Medical Research, \\ Parkville, VIC, Australia; ${ }^{e}$ National Healthcare Group Eye Institute, Tan Tock Seng Hospital, \\ Singapore, Singapore
}

\section{Keywords}

Fingolimod · Uveitis · Relapsing-remitting multiple sclerosis · FTY720 · Rebound syndrome

\begin{abstract}
Fingolimod is an oral sphingosine-1-phosphate (S1P) receptor modulator and the first oral therapy for relapsing-remitting multiple sclerosis. Its use has been complicated by a low rate of cystoid macular edema usually in the first 3 months after commencement of the medication. We report the case of a 34-year-old male with relapsing-remitting multiple sclerosis, who developed acute anterior uveitis on day 5 of fingolimod treatment. He responded to appropriate treatment and cessation of drug, but developed low-grade chronic anterior uveitis without cystoid macular edema. We discuss possible mechanisms of uveitis onset in this group of patients. Urgent ophthalmological review is recommended for patients receiving fingolimod therapy who develop a red, painful eye, which may occur within 5 days of fingolimod treatment initiation.




\section{Case Reports in Ophthalmology}

\section{Introduction}

Fingolimod (FTY720, Gilenya, Novartis Pharmaceuticals, Basel, Switzerland) is the first oral sphingosine-1-phosphate (S1P) receptor modulator therapy found to be effective for relapsing-remitting multiple sclerosis (RRMS) [1-3]. Fingolimod binds to lymphocyte S1P receptors, altering T-cell lymphocyte recirculation by entrapping them in secondary lymphoid organs and reducing infiltration into the central nervous system (CNS). Additional immunomodulatory mechanisms may include influencing the blood-brain barrier function, and interaction with $\mathrm{S}_{1} \mathrm{P}_{1}, \mathrm{~S}_{1} \mathrm{P}_{3}$, and $\mathrm{S}_{1} \mathrm{P}_{5}$ receptors expressed by many CNS neural and nonneural cells $[4,5]$.

In clinical practice, fingolimod is associated with development of cystoid macular edema (CME) in a dose-dependent manner and occurs at a higher rate in patients with a past history of uveitis $[6,7]$. To date, there are no case reports of other forms of ocular inflammation associated with fingolimod; however, uveitis has also been reported in a fingolimod-treated patient who developed varicella zoster virus encephalopathy [8].

\section{Case History}

A 34-year-old male with a 7-year history of RRMS was treated with fingolimod $0.5 \mathrm{mg}$ daily orally. His RRMS was previously stable on monthly infusions of natalizumab (Tysabri, Biogen Idec). However, JC virus testing became positive and treatment was changed to reduce the risk of progressive multifocal leukoencephalopathy. He had a normal ophthalmic examination with best-corrected visual acuity of 20/20 in both eyes and normal screening macular optical coherence scan (OCT) prior to commencing fingolimod. He had no ophthalmic history of ocular involvement with RRMS or any previous history of uveitis. He had received a chickenpox vaccine as a child.

Five days post initiation of treatment, he developed a painful red right eye with blurred vision (Fig. 1) and consulted an ophthalmologist after 14 days of fingolimod treatment. Visual acuity was 20/80 in the right eye and 20/20 in the left eye. The right eye was markedly injected with $1+$ anterior chamber cells and flare, keratic precipitates, and $360^{\circ}$ posterior synechiae. Vitreoretinal examination was normal, with no pars planitis. No CME was found, both clinically and on OCT (Fig. 2). The left eye was unaffected. Syphilis serology, ACE testing, and HLA-B27 testing were negative. Fingolimod was discontinued and dimethyl fumarate was commenced. A tapering regime of topical $1 \%$ prednisolone and $1 \%$ atropine was instituted with good clinical response, albeit mild CME was present on OCT scanning at 6 weeks, which resolved spontaneously (Fig. 2). He re-presented 16 weeks and 6 months after fingolimod cessation with further episodes of mild right anterior uveitis without CME and continued to have further flare-ups of anterior uveitis, eventually developing chronic anterior uveitis, which was controlled with topical $1 \%$ prednisolone daily. At last review, visual acuity was 20/20 in both eyes and no iris atrophy, uveitis, or CME were found. Intraocular pressure was normal throughout his clinical course. His RRMS was stable with no new CNS lesions. 


\section{Case Reports in Ophthalmology}

\section{Discussion}

Our patient developed anterior uveitis without significant CME on day 5 of fingolimod treatment and did not show typical features of herpetic related uveitides. Whilst it is known that bilateral intermediate uveitis does occur in approximately $1 \%$ of patients with MS and between 0.8 and $14 \%$ of uveitis patients have MS $[9,10]$, our patient had no evidence of intermediate uveitis prior to commencing fingolimod or during fingolimod treatment.

The cause of uveitis in patients receiving fingolimod therapy is unknown. One possible mechanism could be a breakdown of the blood-eye barrier in the uvea, similar to the proposed mechanism for development of $\mathrm{CME}$, where agonism of $\mathrm{S}_{1} \mathrm{P}_{1}$ and $\mathrm{S}_{1} \mathrm{P}_{3}$ receptors increases vascular permeability $[11,12]$. Other potential mechanisms include functional agonism of Th1 and Th17 lymphocyte $\mathrm{S}_{1} \mathrm{P}_{1}$ receptors found in patients with multiple sclerosis and uveitis, through activation of herpes virus infection or via fingolimod immunomodulatory activities independent of S1P receptors [13]. Our patient developed unilateral anterior uveitis, in contrast to patients who develop fingolimod-associated CME, which is usually bilateral but can be asymmetrical. Similar to all forms of inflammatory eye disease, the reasons for unilateral presentation are not known.

Our patient developed chronic uveitis, despite ceasing fingolimod at uveitis diagnosis. This may be related to post-fingolimod rebound phenomenon and possibly due to lymphocyte reentry to the uvea or $\mathrm{S}_{1} \mathrm{P}_{1}$ receptor dysmodulation [14], although it should be noted he did not develop clinical post-fingolimod CNS rebound syndrome. Consideration should be given to continuing or ceasing fingolimod, due to the risk of post-fingolimod MS rebound syndrome and some patients may elect to have local uveitis treatment while continuing oral fingolimod.

To our knowledge, this is the first report of acute-on-chronic anterior uveitis, which developed 5 days after initiation of fingolimod treatment in a patient with RRMS and no preexisting uveitis. While the development of uveitis may be a coincidence, it raises the possibility of that fingolimod is pro-inflammatory in the eye and able to cause uveitis in a minority of patients, in addition to the well-described side effect of CME. Patients on fingolimod should be advised of possible adverse visual symptoms and/or pain, which may occur as early as 5 days after treatment initiation [15]. As the $\mathrm{S}_{1} \mathrm{P}_{1}$ receptor is present in the heart, as well as other organs, patients need to be also warned of possible systemic side effects and have appropriate systemic monitoring, particularly at treatment initiation. Ophthalmologists need to consider the role of anterior chamber paracentesis and viral polymerase chain reaction testing in similar patients. We agree with recommendations to undertake ophthalmological examination prior to treatment and suggest urgent ophthalmology review for patients with visual symptoms and/or pain. Further case reports are required to better determine whether there is a causal relationship between fingolimod and acute anterior uveitis in a minority of patients. 
Mack et al.: Acute Anterior Uveitis in a Patient Taking Fingolimod (FTY720) for Multiple Sclerosis

\section{Statement of Ethics}

This case was in accordance with the principles of the Declaration of Helsinki. The patient gave informed consent to its publication.

\section{Disclosure Statement}

The authors have no financial disclosures.

\section{References}

1 Chun J, Hartung HP: Mechanism of action of oral fingolimod (FTY720) in multiple sclerosis. Clin Neuropharmacol 2010;33:91-101.

-2 Cohen JA, Barkhof F, Comi G, et al: Oral fingolimod or intramuscular interferon for relapsing multiple sclerosis. N Engl J Med 2010;362:402-415.

-3 Kappos L, Radue EW, O'Connor P, et al: A placebo-controlled trial of oral fingolimod in relapsing multiple sclerosis. N Engl J Med 2010;362:387-401.

4 Horga A, Montalban X: FTY720 (fingolimod) for relapsing multiple sclerosis. Expert Rev Neurother 2008;8:699-714.

5 Groves A, Kihara Y, Chun J: Fingolimod: direct CNS effects of sphingosine 1-phosphate (S1P) receptor modulation and implications in multiple sclerosis therapy. J Neurol Sci 2013;328:9-18.

-6 Jain N, Bhatti MT: Fingolimod-associated macular edema: incidence, detection, and management. Neurology 2012;78:672-680.

7 Zarbin MA, Jampol LM, Jager RD, et al: Ophthalmic evaluations in clinical studies of fingolimod (FTY720) in multiple sclerosis. Ophthalmology 2013;120:1432-1439.

$>8$ Issa NP, Hentati A: VZV encephalitis that developed in an immunised patient during fingolimod therapy. Neurology 2015;84:99-100.

9 Burkholder BM, Dunn JP: Multiple sclerosis-associated uveitis. Expert Rev Ophthalmol 2013;7:587594.

10 Hildebrandt AL, Mackensen F: Uveitis in multiple sclerosis: overview and perspectives. Ophthalmologe 2014;111:733-739.

11 Rotstein NP, Miranda GE, Abrahan CE, et al: Regulating survival and development in the retina: key roles for simple sphingolipids. J Lipid Res 2010;51:1247-1262.

12 Brinkmann V, Baumruker T: Pulmonary and vascular pharmacology of sphingosine 1-phosphate. Curr Opin Pharmacol 2006;6:244-250.

13 Chi H: Sphingosine 1-phosphate and immune regulation: trafficking and beyond. Trends Pharmacol Sci 2011;32:16-24

14 Cavone L, Felici R, Lapucci A, et al: Dysregulation of sphingosine 1 phosphate receptor-1 (S1P1) signaling and regulatory lymphocyte-dependent immunosuppression in a model of post-fingolimod MS rebound. Brain Behav Immun 2015;50:78-86.

15 Liu L, Cuthbertson F: Early bilateral cystoid macular oedema secondary to fingolimod in multiple sclerosis. Case Rep Med 2012;2012:134636. 


\section{Case Reports in Ophthalmology}

\begin{tabular}{l|l}
\hline Case Rep Ophthalmol 2016;7:284-288 \\
\hline DOI: 10.1159/000453392 & $\begin{array}{l}\text { @ 2016 The Author(s). Published by S. Karger AG, Basel } \\
\text { www.karger.com/cop }\end{array}$ \\
\hline
\end{tabular}

Mack et al.: Acute Anterior Uveitis in a Patient Taking Fingolimod (FTY720) for Multiple Sclerosis

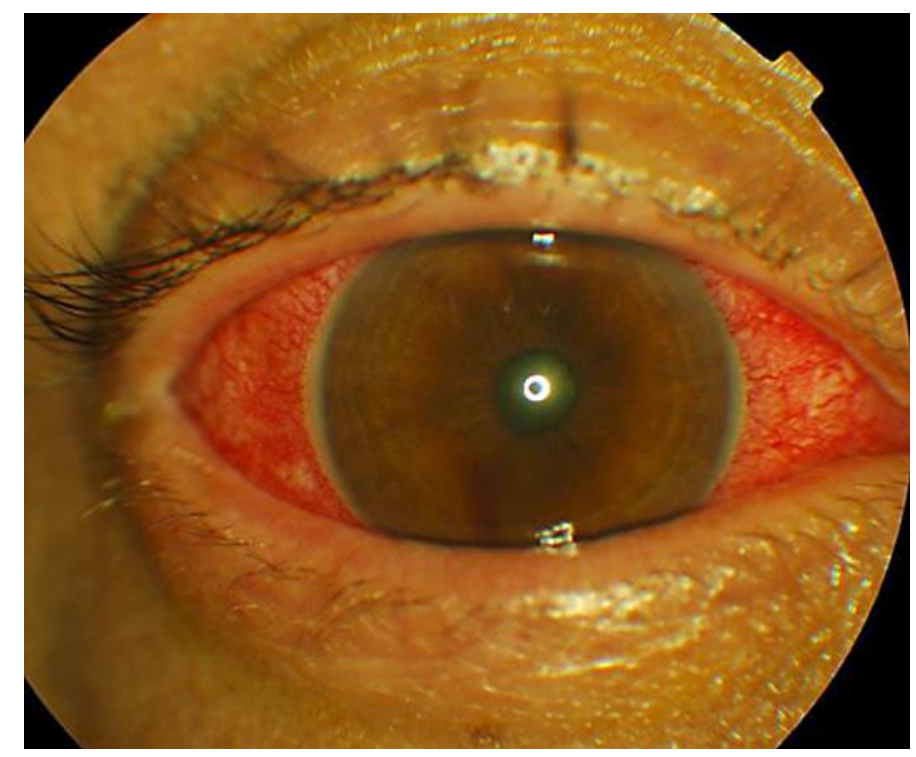

Fig. 1. Right eye at presentation 14 days after initiation of fingolimod treatment for relapsing-remitting multiple sclerosis demonstrating conjunctival injection, constricted pupil, and lack of purulent discharge.
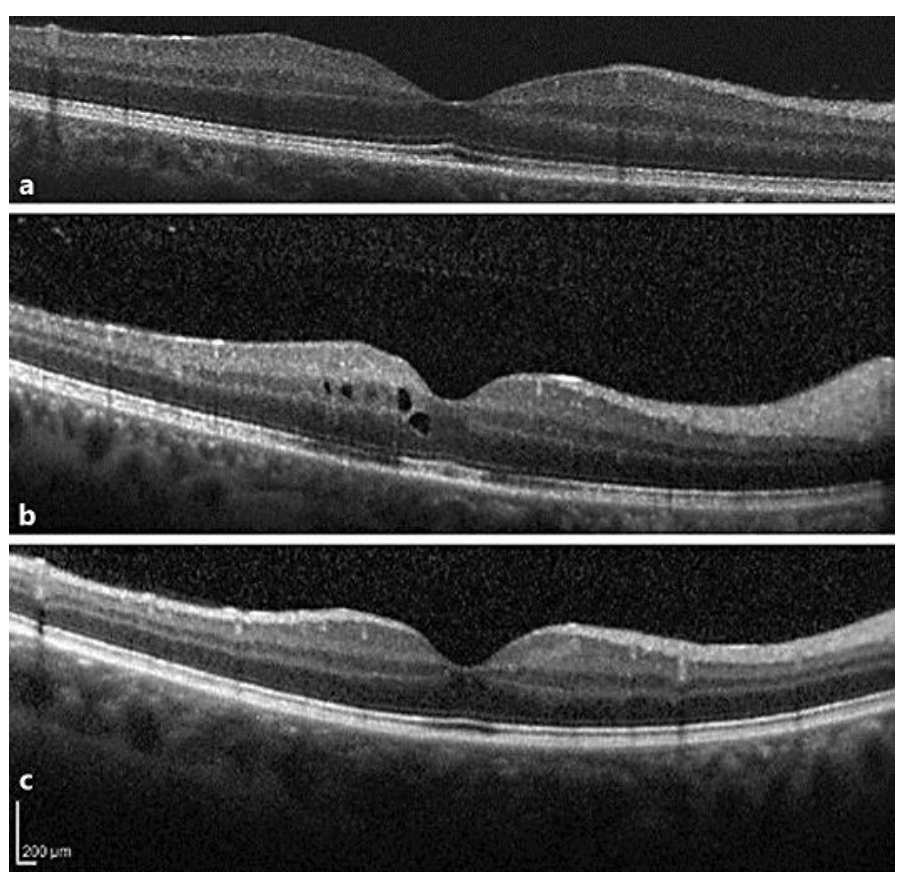

Fig. 2. Right-eye macular optical coherence scan (Spectralis, Heidelberg Engineering) in a patient who developed acute anterior uveitis after treatment with fingolimod for relapsing-remitting multiple sclerosis. a At 14 days, when fingolimod was discontinued. b At 6 weeks, demonstrating cystoid macular edema and vitreous cells. c At 16 weeks. 